\title{
Three-loop massive scalar effective potential
}

\section{O. L. Veretin*}

II. Institut für Theoretische Physik, Universität Hamburg, Luruper Chaussee 149, 22761 Hamburg, Germany

E-mail: veretin@mail.desy.de

\section{B. A. Kniehl}

II. Institut für Theoretische Physik, Universität Hamburg, Luruper Chaussee 149, 22761 Hamburg, Germany

E-mail: kniehl@mail.desy.de

\section{A. F. Pikelner}

II. Institut für Theoretische Physik, Universität Hamburg, Luruper Chaussee 149, 22761 Hamburg, Germany,

E-mail: andrey.pikelner@desy.de

We evaluate analytically the three-loop effective potential in theories with one Higgs boson and several Goldstone bosons. The results are applicable to the scalar sector of the Standard Model. Using the technique of differential equations we express all master integrals in terms of cyclotomic polylogarithms.

Loops and Legs in Quantum Field Theory (LL2018)

29 April 2018 - 04 May 2018

St. Goar, Germany

${ }^{*}$ Speaker. 


\section{Introduction}

The effective potential $[1,2]$ plays a very important role in investigating spontaneous symmetry breaking. In the Standard Model it was a topic of numerous studies over the years $[3,4,5,6,7]$ with partial results at three [8] and four-loop order [9]. The analysis of the effective potential at the two-loop order brings us to the conclusion that the electroweak vacuum can be at least metastable up to very high energies of the order of Planck scale $[10,11,12]$. Such analysis at the three-loop order would require apart from four-loop renormalization group functions [13, 14, 15, 16, 17, 18] also the matching conditions, which are known only up to the two-loop order [19]. The important step, however, is the recent result for a general renormalizable theory [20], with loop-integrals known numerically $[21,22,23]$.

In this work we consider the pure scalar sector of a general renormalizable theory and evaluate the effective potential up to three loops analytically. The integrals that appear in our calcualtion can have up to two different mass scales. In the case of $\mathscr{O}(n)$ symmetry we reproduce the scalar $\varphi^{4}$ theory with spontaneous symmetry breaking. This theory is a matter of interest for the theory of the phase transitions and has been calculated in the series of papers [24, 25]. The results presented here reproduce the contribution of the scalar sector of the Standard Model to the effective potential.

\section{Effective potential in the scalar theory and the three-loop master integrals}

Consider the scalar potential constructed from one Higgs field $H$ and several Goldstone fields $G_{i}$

$$
\mathscr{V}_{S}=\mu_{1} H^{2}+\mu_{2} G_{i}^{2}+\frac{\tau_{0}}{6} H^{3}+\frac{\tau_{i}}{6} H G_{i}^{2}+\frac{\lambda_{0}}{24} H^{4}+\frac{\lambda_{i}}{12} H^{2} G_{i}^{2}+\frac{\lambda_{i j}}{24} G_{i}^{2} G_{j}^{2},
$$

where $\tau_{i}, \lambda_{i}$ and $\lambda_{i j}$ are couplings. This scalar potential can describe as well some scalar theories (e.g. $O(n)$ scalar model with spontaneous symmetry breaking) as the Standard Model. In the Standard Model in the broken phase the parameters are expressed as

$$
\tau_{0}=\tau_{i}=\lambda \phi, \quad \lambda_{0}=\lambda_{i}=\lambda_{i j}=\lambda, \quad \mu_{1}=m^{2}+\frac{\lambda}{2} \phi^{2}, \quad \mu_{2}=m^{2}+\frac{\lambda}{6} \phi^{2},
$$

where $\lambda$ is the scalar self-coupling, $\phi$ is the vacuum expectation value and $m$ is the mass parameter of the theory.

In the calculation of the potential, given by (2.1), at the three-loop level we should consider three-loop vacuum Feynman integrals with two different masses. All integrals which appear in this calculation can be mapped depending on the masses on internal lines on one of the three auxiliary topologies $J_{A}, J_{B}$ and $J_{C}$ shown in Fig. 1 . We set $m_{2}=1$ and denote the ratio $x=m_{1}^{2} / m_{2}^{2}$. After this rescaling we write

$$
\begin{aligned}
& J_{n_{1} \ldots n_{6}}^{A}=\int \frac{d\left[k_{1}\right] d\left[k_{2}\right] d\left[k_{3}\right]}{\left(k_{1}^{2}+1\right)^{n_{1}}\left(k_{2}^{2}+1\right)^{n_{2}}\left(k_{3}^{2}+1\right)^{n_{3}}\left(k_{12}^{2}+1\right)^{n_{4}}\left(k_{23}^{2}+1\right)^{n_{5}}\left(k_{31}^{2}+1\right)^{n_{6}}}, \\
& J_{n_{1} \ldots n_{6}}^{B}=\int \frac{d\left[k_{1}\right] d\left[k_{2}\right] d\left[k_{3}\right]}{\left(k_{1}^{2}+x\right)^{n_{1}}\left(k_{2}^{2}+x\right)^{n_{2}}\left(k_{3}^{2}+x\right)^{n_{3}}\left(k_{12}^{2}+1\right)^{n_{4}}\left(k_{23}^{2}+1\right)^{n_{5}}\left(k_{31}^{2}+1\right)^{n_{6}}}, \\
& J_{n_{1} \ldots n_{6}}^{C}=\int \frac{d\left[k_{1}\right] d\left[k_{2}\right] d\left[k_{3}\right]}{\left(k_{1}^{2}+1\right)^{n_{1}}\left(k_{2}^{2}+x\right)^{n_{2}}\left(k_{3}^{2}+x\right)^{n_{3}}\left(k_{12}^{2}+x\right)^{n_{4}}\left(k_{23}^{2}+1\right)^{n_{5}}\left(k_{31}^{2}+x\right)^{n_{6}}},
\end{aligned}
$$


where $k_{a b}=k_{a}-k_{b}$ and loop integration measure $d\left[k_{i}\right]=d^{d} k / \pi^{d / 2} e^{\varepsilon \gamma_{E}}$, with $d=4-2 \varepsilon$ being the dimension of the space-time and $\gamma_{E}$ is the Euler constant.

The integrals from the topology $\mathbf{A}$ are single scale integrals and are known for a long time [26]. Recently they have been evaluated up to weight six [27].

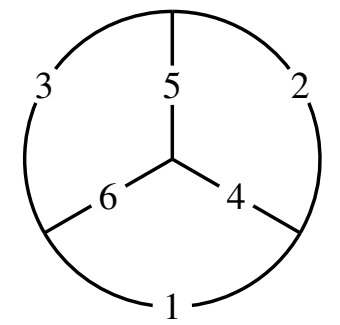

A

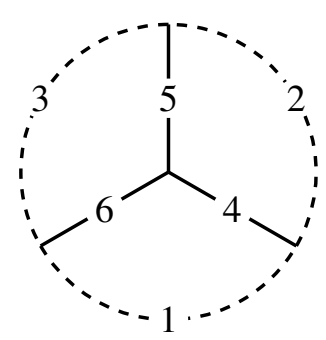

B

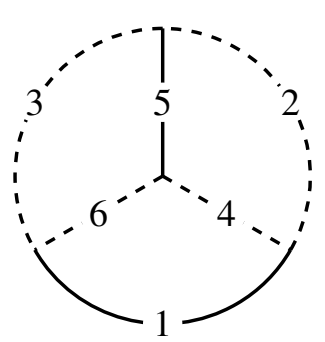

C

Figure 1: Master topologies. Solid lines have masses equal to one and dashed lines - mass squared equal to $x$.

The other two topologies $\mathbf{B}$ and $\mathbf{C}$ have two mass scales and depend on the ratio $x$. For each of these topologies we have constructed a set of reduction rules with the help of LiteRed package [28] and identified the set of the master integrals. Differentiating with respect to $x$ all the master integrals and reducing the right hand sides to the set of master integrals we obtain the systems of differential equations.

$$
\frac{\partial J_{i}^{\mathrm{b}}}{\partial x}=M_{i j}^{\mathrm{b}} J_{j}^{\mathrm{b}}, \quad \frac{\partial J_{i}^{\mathrm{c}}}{\partial x}=M_{i j}^{\mathrm{c}} J_{j}^{\mathrm{c}} .
$$

The elements of the matrices $M^{\mathrm{b}}$ and $M^{\mathrm{c}}$ are rational functions of the mass ratio $x$ and the space time dimension $d$. It is convenient to switch from the original basis $\left\{J^{\mathrm{b}}, J^{\mathrm{c}}\right\}$ to the new canonical basis $\left\{g^{\mathrm{b}}, g^{\mathrm{c}}\right\}$ of the master integrals introduced in [29]. The integrals in the canonical basis have uniform transcendentality weights of the coefficients of their expansion in $\varepsilon$ and the matrix of the differential equation have the special so called $\varepsilon$-form.

To find the explicit form of the transformation matrices and the matrix of the differential equations in the $\varepsilon$-form we use public tools Fuchsia [30] and CANONICA [31]. Both of these tools implement different algorithms, which rely heavily on the polynomial rational form of the transformation matrices. Our matrices become rational after the following change of variable

$$
x=\frac{y^{2}}{\left(1+y^{2}\right)^{2}},
$$

after which we can successfully find the transformation matrices $T$ and the corresponding DE systems take the form

$$
\frac{\partial g_{i}^{\mathrm{b}}}{\partial y}=\varepsilon B_{i j} g_{j}^{\mathrm{b}}, \quad \frac{\partial g_{i}^{\mathrm{c}}}{\partial y}=\varepsilon C_{i j} g_{j}^{\mathrm{c}}
$$

Now the matrices $B$ and $C$ do not depend on the space-time dimension $d$. We can proceed further and decompose them into the sums of the constant matrices with all dependence on the kinematical 
vairable $y$ factorized out. These decompositions read

$$
\begin{aligned}
B(y)= & \left(f_{0}^{0} B_{0,0}+f_{1}^{0} B_{1,0}+f_{2}^{0} B_{2,0}+f_{3}^{0} B_{3,0}+f_{3}^{1} B_{3,1}\right. \\
& \left.+f_{4}^{1} B_{4,1}+f_{6}^{0} B_{6,0}+f_{6}^{1} B_{6,1}+f_{12}^{1} B_{12,1}+f_{12}^{3} B_{12,3}\right) \\
C(y)= & \left(f_{0}^{0} C_{0,0}+f_{1}^{0} C_{1,0}+f_{2}^{0} C_{2,0}+f_{3}^{0} C_{3,0}+f_{3}^{1} C_{3,1}+f_{4}^{1} C_{4,1}+f_{6}^{0} C_{6,0}+f_{6}^{1} C_{6,1}+f_{8}^{3} C_{8,3}\right)
\end{aligned}
$$

The matrices $B_{a, b}$ and $C_{a, b}$ are pure numerical (rational numbers) and all dependence on $y$ is hidden in the functions $f_{a}^{b}(y)$, defined as

$$
f_{0}^{0}(y)=\frac{1}{y}, \quad f_{a}^{b}(y)=\frac{y^{b}}{\Phi_{a}(y)}
$$

where $\Phi_{n}(y)$ denotes the $n$-th cyclotomic polynomial. In our calculation the following cyclotomic polynomials appear

$$
\begin{aligned}
& \Phi_{1}=y-1, \\
& \Phi_{2}=y+1, \\
& \Phi_{3}=y^{2}+y+1, \\
& \Phi_{4}=y^{2}+1, \\
& \Phi_{6}=y^{2}-y+1 \\
& \Phi_{8}=y^{4}+1, \\
& \Phi_{12}=y^{4}-y^{2}+1 .
\end{aligned}
$$

Such functions have a nice property to have the transparent integration rules leading to the special type of functions representing the iterated integrals generalizing harmonic polylogarithms. Such functions are cyclotomic harmonic polylogarithms and were introduced earlier in [32].

The system of differential equations in the $\varepsilon$-form has a nice property that after expansion of all master integrals in $\varepsilon$, DE's for the series coefficient completely decouple and can be written in the form

$$
g_{i}^{\mathrm{b}}\left\{\varepsilon^{n}\right\}(y)=\int_{0}^{1} d y B_{i j} g_{i}^{\mathrm{b}}\left\{\varepsilon^{n-1}\right\}(y)+\mathscr{C}_{i, n},
$$

where $g\left\{\varepsilon^{n}\right\}(y)$ denotes the $O\left(\varepsilon^{n}\right)$ coefficient of the $\varepsilon$-expansion of $g(y)$. Using (2.9) we can construct the iterative integrals of functions (2.11). Starting from the lowest order of expansion in $\varepsilon$, which is just a constant, we can integrate order by order using the rule (2.19) and obtain the following definition of CHPL

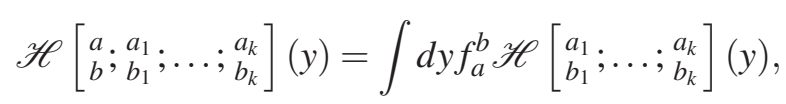

with $\mathscr{H}[](y)=1$.

At each step of integration we need to fix an integration constant. This is done using the expansions of the integrals in the small $x$ aproximation. These expansions are not naive and contain on top of power like terms also logarithms due to subgraphs. 


\section{Conclusions}

The functions, defined in (2.20), were enough to find all the master integrals and present the three-loop effective potential (2.1) in the analytic form.

At high energy scales the strength of $\phi$ is much higher than the mass parameter $m$ of the lagrangian and we can neglect it in the formulae (2.2). Thus the ratio $x=m_{1}^{2} / m_{2}^{2}$ is equal to $1 / 3$. This limit corresponds to the value $y=e^{-i \pi / 6}$. For this massles version of the theory (2.1) some partial results are known $[33,34]$. We have checked whether the expressions for all master integrals at this point can be expressed in terms of the basis constructed in [27] or more general basis of sixht root of unity [35]. The result is that at weight two it is still possible (this has been achived already in $[33,34])$, while at higher weights it is not possible. Looking at the cyclotomic polynomials one can expect that the basis, constructed from the twelveth root of unity would be apropriate.

In the present work we have evaluated the thee-loop effective potential in the scalar sector analytically. The result is expressed in terms of the cyclotomic polylogarims of degrees 1,2,3,4,6,8, and 12 up to the weight six. The general three loop-vacuum master integral with two different mass scales does not apparently lie in the class of polylogarithmic functions, while the particular subset, relevant to the scalar sector does.

\section{References}

[1] S. R. Coleman, E. J. Weinberg, Radiative Corrections as the Origin of Spontaneous Symmetry Breaking, Phys. Rev. D7 (1973) 1888-1910.

[2] R. Jackiw, Functional evaluation of the effective potential, Phys. Rev. D9 (1974) 1686.

[3] M. Sher, Electroweak Higgs Potentials and Vacuum Stability, Phys. Rept. 179 (1989) 273-418.

[4] M. Lindner, M. Sher, H. W. Zaglauer, Probing Vacuum Stability Bounds at the Fermilab Collider, Phys. Lett. B228 (1989) 139-143.

[5] P. B. Arnold, S. Vokos, Instability of hot electroweak theory: bounds on $\mathrm{m}(\mathrm{H})$ and $\mathrm{M}(\mathrm{t})$, Phys. Rev. D44 (1991) 3620-3627.

[6] C. Ford, D. R. T. Jones, P. W. Stephenson, M. B. Einhorn, The Effective potential and the renormalization group, Nucl. Phys. B395 (1993) 17-34.

[7] C. Ford, I. Jack, D. R. T. Jones, The Standard model effective potential at two loops, Nucl. Phys. B387 (1992) 373-390, [Erratum: Nucl. Phys.B504,551(1997)].

[8] S. P. Martin, Three-loop Standard Model effective potential at leading order in strong and top Yukawa couplings, Phys. Rev. D89 (1) (2014) 013003.

[9] S. P. Martin, Four-Loop Standard Model Effective Potential at Leading Order in QCD, Phys. Rev. D92 (5) (2015) 054029.

[10] A. V. Bednyakov, B. A. Kniehl, A. F. Pikelner, O. L. Veretin, Stability of the Electroweak Vacuum: Gauge Independence and Advanced Precision, Phys. Rev. Lett. 115 (20) (2015) 201802.

[11] D. Buttazzo, G. Degrassi, P. P. Giardino, G. F. Giudice, F. Sala, A. Salvio, A. Strumia, Investigating the near-criticality of the Higgs boson, JHEP 12 (2013) 089.

[12] F. Bezrukov, M. Yu. Kalmykov, B. A. Kniehl, M. Shaposhnikov, Higgs Boson Mass and New Physics, JHEP 10 (2012) 140, [,275(2012)]. 
[13] A. V. Bednyakov, A. F. Pikelner, V. N. Velizhanin, Anomalous dimensions of gauge fields and gauge coupling beta-functions in the Standard Model at three loops, JHEP 01 (2013) 017.

[14] A. V. Bednyakov, A. F. Pikelner, V. N. Velizhanin, Yukawa coupling beta-functions in the Standard Model at three loops, Phys. Lett. B722 (2013) 336-340.

[15] A. V. Bednyakov, A. F. Pikelner, V. N. Velizhanin, Higgs self-coupling beta-function in the Standard Model at three loops, Nucl. Phys. B875 (2013) 552-565.

[16] A. V. Bednyakov, A. F. Pikelner, Four-loop strong coupling beta-function in the Standard Model, Phys. Lett. B762 (2016) 151-156.

[17] M. F. Zoller, Top-Yukawa effects on the beta-function of the strong coupling in the SM at four-loop level, JHEP 02 (2016) 095.

[18] K. G. Chetyrkin, M. F. Zoller, Leading QCD-induced four-loop contributions to the beta-function of the Higgs self-coupling in the SM and vacuum stability, JHEP 06 (2016) 175.

[19] B. A. Kniehl, A. F. Pikelner, O. L. Veretin, Two-loop electroweak threshold corrections in the Standard Model, Nucl. Phys. B896 (2015) 19-51.

[20] S. P. Martin, Effective potential at three loops, Phys. Rev. D96 (9) (2017) 096005.

[21] S. P. Martin, D. G. Robertson, Evaluation of the general 3-loop vacuum Feynman integral, Phys. Rev. D95 (1) (2017) 016008.

[22] A. Freitas, “Three-loop vacuum integrals with arbitrary masses," JHEP 1611 (2016) 145 doi:10.1007/JHEP11(2016)145 [arXiv:1609.09159 [hep-ph]].

[23] S. Bauberger and A. Freitas, "TVID: Three-loop Vacuum Integrals from Dispersion relations," arXiv:1702.02996 [hep-ph].

[24] C. Ford, D. R. T. Jones, The Effective potential and the differential equations method for Feynman integrals, Phys. Lett. B274 (1992) 409-414, [Erratum: Phys. Lett.B285,399(1992)].

[25] J. M. Chung, B. K. Chung, Three loop renormalization of the effective potential, Phys. Rev. D56 (1997) 6508-6523, [Erratum: Phys. Rev.D59,109902(1999)].

[26] M. Steinhauser, MATAD: A Program package for the computation of MAssive TADpoles, Comput. Phys. Commun. 134 (2001) 335-364.

[27] B. A. Kniehl, A. F. Pikelner, O. L. Veretin, Three-loop massive tadpoles and polylogarithms through weight six, JHEP 08 (2017) 024.

[28] R. N. Lee, “Presenting LiteRed: a tool for the Loop InTEgrals REDuction,” arXiv:1212.2685 [hep-ph].

[29] J. M. Henn, Multiloop integrals in dimensional regularization made simple, Phys. Rev. Lett. 110 (2013) 251601

[30] O. Gituliar, V. Magerya, Fuchsia: a tool for reducing differential equations for Feynman master integrals to epsilon form, Comput. Phys. Commun. 219 (2017) 329-338.

[31] C. Meyer, Algorithmic transformation of multi-loop master integrals to a canonical basis with CANONICA, Comput. Phys. Commun. 222 (2018) 295-312.

[32] J. Ablinger, J. Blumlein, C. Schneider, Harmonic Sums and Polylogarithms Generated by Cyclotomic Polynomials, J. Math. Phys. 52 (2011) 102301. 
[33] J. M. Chung, B. K. Chung, Calculation of a class of three loop vacuum diagrams with two different mass values, Phys. Rev. D59 (1999) 105014.

[34] A. V. Kotikov, Compact analytical form for a class of three loop vacuum Feynman diagrams, JHEP 09 (1998) 001.

[35] J. M. Henn, A. V. Smirnov and V. A. Smirnov, Nucl. Phys. B 919 (2017) 315 doi:10.1016/j.nuclphysb.2017.03.026 [arXiv:1512.08389 [hep-th]]. 